\section{A SIAH of K-RAS relief}

\section{By Kai-Jye Lou, Staff Writer}

Researchers at the Mayo Clinic College of Medicine have shown that inhibiting the activity of an E3 ubiquitin ligase can inhibit cell proliferation and increase apoptosis across various types of lung and pancreatic cancers. ${ }^{1}$ The most dramatic effects were in aggressive cancer cell lines driven by activating RAS mutations, which tend to be resistant to marketed drugs and radiotherapy. Targeting the ligase in vivo will be challenging, however.

The target, seven in absentia homolog 2 (SIAH2), is a downstream component in the mammalian RAS signaling pathway and is upregulated in cancer cells compared with in normal cells. Previous work had suggested that SIAH2 has substrates that can affect more upstream components like v-raf-1 murine leukemia (RAF) and mitogen-activated protein kinases (MAPKs) through negative-feedback regulation. ${ }^{2,3}$

E3 ubiquitin ligases mark substrates for proteolytic degradation via the ubiquitin-proteasome pathway ( see Figure 1, "Regulation of the RAS signaling pathway through SIAH2").

Aberrant signaling in components of the mammalian RAS pathway is known to be a driving factor in many forms of cancer. Moreover, activating mutations in the RAS genes themselves, most notably in $\mathrm{v}$-Kiras2 Kirsten rat sarcoma viral oncogene homolog (K-RAS), can confer aggressive cancer phenotypes that exhibit rapid proliferation and drug resistance.

In a paper published in the Journal of the National Cancer Institute, the Mayo Clinic group, led by Amy Tang, assistant professor in the Department of Surgery and the Department of Biochemistry and Molecular Biology, studied small hairpin RNA knockdown of SIAH2 in various cultured human lung carcinomas, including the K-RAS-mutated A549 subtype. The team found that the knockdown increased apoptosis and inhibited cell proliferation compared with control cells that received mock or SIAH1-targeting shRNA.

Similar results were seen in the cells when overexpression of a proteolysis-deficient SIAH2 mutant was used to knock down SIAH2 activity. Also, nude mice injected with carcinoma cells overexpressing the SIAH2 mutant or treated with SIAH2-targeting shRNA had less tumor growth than mice that received carcinomas overexpressing control GFP or mice treated with mock shRNA.

"The novelty of our findings is that we approach the problem by inhibiting the RAS pathway at the level of the most downstream component rather than at the top," said Tang. "Signal transduction pathways in cancer are complex and interact with numerous pathways. Inhibition of a cell-surface receptor, such as EGFR [epidermal growth factor receptor], can reduce signaling in the RAS pathway. However, other pathways can compensate for this and still contribute to tumorigenesis."

Tang told SciBX that the efficacy of SIAH2 inhibition seems to scale up with the aggressiveness and proliferative capacity of the targeted cancer cells, with the greatest effects being observed in lung cancer subtypes with hyperactivated EGFR and RAS signaling.

"So far, we have been able to inhibit tumor growth in pancreatic cancer, lung cancer and several other cancer types-and this includes some of the most aggressive cancer cell lines like A549," she said.

"Many of the current targeted therapies do not work in K-RAS mutant tumors. Anything that would cripple RAS signaling would be of great clinical significance," said Ronald DePinho, director of the Belfer Institute for Applied Cancer Science at the Dana-Farber Cancer Institute and a professor of medicine and genetics at the institute and at Harvard Medical School.

Data presented at the American Society of Clinical Oncology meeting in June showed that K-RAS genetic status is essential for determining whether to treat metastatic colorectal cancer patients with EGFR inhibitors. For example, multiple abstracts correlated the efficacy of Erbitux cetuximab with wild-type K-RAS, including a retrospective analysis of the Phase III CRYSTAL (Cetuximab Combined with Irinotecan in First Line Therapy for Metastatic Colorectal Cancer) study showing that patients with mutated $K$-RAS receiving Erbitux plus chemotherapy did not show improved progression-free survival (PFS) compared with patients receiving chemotherapy alone. ${ }^{4}$

Last month, the National Comprehensive Cancer Network updated its colon and rectal cancer clinical practice guidelines to recommend determination of $K-R A S$ gene status and the use of Erbitux and Vectibix panitumumab only in patients with tumors characterized by the wildtype gene. Previously, K-RAS testing had not been included in the guidelines.

Erbitux is marketed by ImClone Systems Inc. (now part of Eli Lilly and Co.), Bristol-Myers Squibb Co. and Merck KGaA to treat colorectal cancer and squamous cell carcinoma of the head and neck (SCCHN). Vectibix, from Amgen Inc. and Takeda Pharmaceutical Co. Ltd., is marketed to treat colorectal cancer.

David Reese, head of global development for Vectibix at Amgen, told SciBX that "patients with $K-R A S$ mutant tumors are a population with unmet medical needs-so any potential therapeutics that target components in this pathway and show efficacy in this population of patients would be of great interest."

He said the results published in JNCI were "intriguing, but this is early data that needs to be validated and reproduced in additional in vivo and in vitro models."

Regardless of how SIAH2 is targeted, Murray Robinson, SVP of oncology at Aveo Pharmaceuticals Inc., wanted to see data in additional in vivo cancer models.

Aveo's lead compound is AV-951, an inhibitor of VEGF receptor 1, VEGF receptor 2 and VEGF receptor 3. It is in a Phase II trial to treat metastatic renal cell carcinoma. 


\section{TARGETS \& MECHANISMS}

Figure 1. Regulation of the RAS signaling pathway through SIAH2. A Journal of the National Cancer Institute paper shows that inducing a seven in absentia homolog 2 (SIAH2) deficiency in RASmutated lung cancer strongly inhibited mitogen-activated protein kinase (MAPK) in the RAS signaling pathway and resulted in decreased cell proliferation and increased apoptosis. ${ }^{1}$ Amy Tang, lead author on the paper, thinks an unidentified substrate of SIAH2, an E3 ubiquitin ligase, is involved in increased cell proliferation via MAPK activation [a]. Thus, she believes that degradation of the substrate by interactions with SIAH2 leads to the inhibition of MAPK seen in the recent study. V-raf-1 murine leukemia (RAF) and mitogen-activated protein kinase kinase (MAP2K; MEK) also play a role in Ras signalling.

It is not yet clear whether the substrate interacts

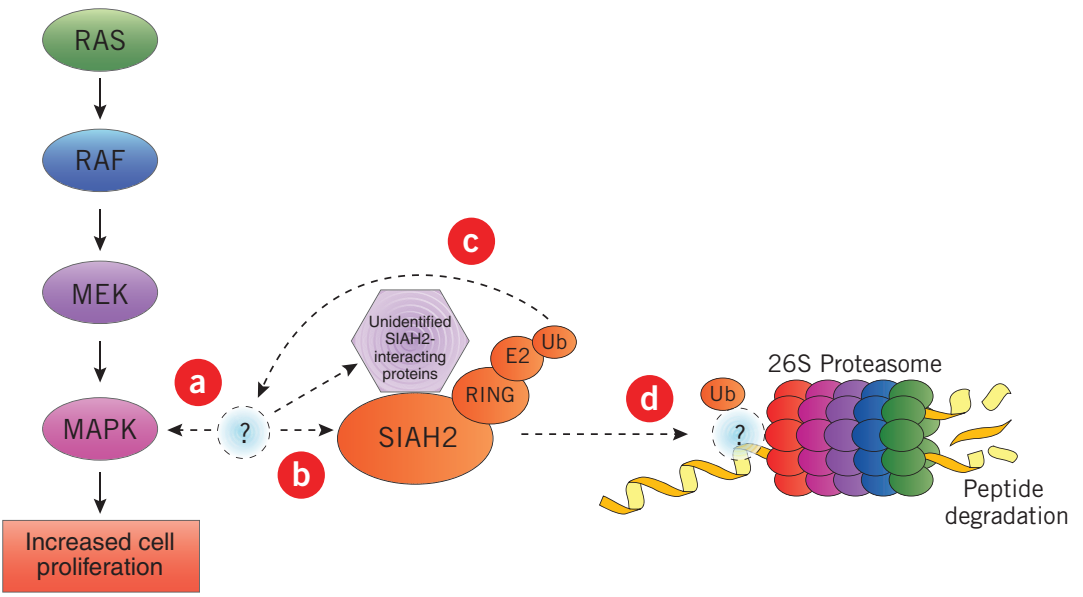
with SIAH2 directly or through other proteins that interact with $\mathrm{SIAH} 2$ [b], but Tang believes that a ubiquitin-conjugating enzyme (E2) interacting with the RING finger domain of SIAH2 is able to transfer ubiquitin $(\mathrm{Ub})$ to the unidentified substrate [c]. She thinks the ubiquitinated SIAH2 substrate is then targeted to the $26 \mathrm{~S}$ proteasome, where it undergoes proteolysis [d].

Whatever the mechanism, the results reported in $\mathrm{JNCl}$ suggest that compounds that directly or indirectly lower SIAH2 activity on the substrate could be useful for treating cancer. Dotted lines denote that such interactions still need to be conclusively demonstrated.

Similarly, DePinho and Jeff Miner, senior director of inflammation and oncology at Ardea Biosciences Inc., wanted to see what happens when SIAH2 is knocked down in an animal model of cancer. Miner also said it would be interesting to compare SIAH2 knockdown with the effects of inhibitors of mitogen-activated protein kinase kinase (MAP2K; $\mathrm{MEK})$, an important target in the RAS signaling pathway.

Ardea has two MEK inhibitors in development. RDEA119 is in a Phase I/II trial to treat advanced cancer. RDEA436, a second-generation molecule, is expected to begin a Phase I trial in inflammatory disease in 1 Q09.

Jüergen Hess, manager of scientific affairs at Trion Pharma GmbH, said, "SIAH2 represents an interesting cancer target structure for further preclinical validation. Nevertheless, the R\&D activities should be broadened to support and supplement the current understanding of SIAH2 function in tumor proliferation and defense."

In particular, he wanted to see additional work to uncover the underlying mechanisms by which SIAH2 knockdown kills lung cancer cells. Hess also said more histological data from cancer patients and healthy controls should be collected to further validate SIAH2's tumor tissue specificity.

Trion's Rexomun ertumaxomab, an antibody targeting HER2 and CD3, is in Phase II testing to treat metastatic breast cancer.

\section{Big challenges for small molecules}

Raymond Deshaies, a Howard Hughes Medical Institute investigator and a professor of biology at the California Institute of Technology, Robinson and DePinho all want to see proof-of-concept studies showing that small molecules can inhibit SIAH2 activity.
"It's one thing to do this with molecular genetic tools, but that can be a long way from getting something that can move forward into the clinic," Robinson said.

Indeed, actually hitting the E3 ubiquitin ligase is likely to be a challenge. Despite a flurry of interest about a decade ago, there are no FDA-approved compounds targeting the activity of E3 ubiquitin ligases. Only a handful of companies, including Roche and Takeda's Millennium Pharmaceuticals Inc. unit, still have active clinical-stage programs in the area.

"It's one thing to do this
with molecular genetic
tools, but that can be a
long way from getting
something that can move
forward into the clinic."
-Murray Robinson,
Aveo Pharmaceuticals Inc.

Roche's R7112, a small molecule antagonist of $\mathrm{Mdm} 2 \mathrm{p} 53$ binding protein homolog (mouse) (MDM2), is in Phase I testing to treat solid tumors and hematologic malignancies. Like SIAH2, MDM2 is an E3 ubiquitin ligase.

Millennium's MLN4924 small molecule inhibitor of NEDD 8 activating enzyme E1 subunit 1 (NAE1) is in Phase I testing for cancer. NAE is not an E3 ubiquitin ligase, but it regulates an upstream event needed to activate an E3 ligase.

Part of the problem is the relatively limited understanding of E3 ubiquitin ligase reaction mechanisms. Unlike classical enzymes that tend to have discreet catalytic sites that serve as prime targets for small molecule inhibition, the ligases' ubiquitination reaction seems to rely heavily on protein-protein interactions.

"Protein-protein interactions come in many forms, and many have large surfaces of interaction such that two interacting proteins can have multiple contact points-these are difficult to block with small molecule inhibitors," said Robinson.

"A number of companies have halted their efforts and moved on to 


\section{TARGETS \& MECHANISMS}

other proteasome regulatory proteins, like NEDD8," said Miner. “Other companies continue to work on targeting E3 ligases; however, this class of targets has not been clinically validated."

Using larger molecules such as antibodies presents its own set of problems, as SIAH2 is an intracellular target.

DePinho, however, noted that early work targeting the proteasome was met with similar levels of skepticism. "Look at proteasome inhibitors-when research in this area began, most thought that targeting the proteasome would not be feasible," he said. "Now we have drugs like Velcade that do just that."

Velcade bortezomib, a small molecule dipeptide boronic acid proteasome inhibitor, is marketed by Millennium and Johnson \& Johnson to treat multiple myeloma and mantle cell lymphoma.

Tang acknowledged that screening for a small molecule E3 ligase inhibitor could be a formidable challenge, but noted that Roche's progress with R7112 shows that a clinical-stage small molecule candidate is achievable.

Deshaies did suggest possible alternatives to developing small molecules that directly target SIAH2. These include inhibitors that prevent SIAH2 from interacting with its ubiquitin-conjugating enzyme (E2). Another option, he said, would be to identify post-translational modifications-like phosphorylation or conjugation with ubiquitin or a related molecule - that SIAH2 relies on for its activity.

Miner thinks inhibition of SIAH2 activity has the potential to show synergy with inhibitors of MEK, Raf and kinases in other pathways.

Tang's next goal is to find out whether knocking down SIAH2 activity blocks or reverses the progression of established tumors. Also, she said her group is trying to identify additional components along the path- way by which SIAH2 inhibition negatively regulates MAPK in the RAS pathway.

The work reported in the JNCI article is not patented.

Lou, K.-J. SciBX 1(44); doi:10.1038/scibx.2008.1063

Published online Dec. 11, 2008

\section{REFERENCES}

1. Ahmed, A.U. et al. J. Natl. Cancer Inst.; published online Nov. 11, 2008; doi:10.1093/jnci/djn365

Contact: Amy H. Tang, Mayo Clinic College of Medicine, Rochester, Minn. e-mail: tang.amy@mayo.edu

2. Schmidt, R.L. et. al. Cancer Res. 67, 11798-11810 (2007)

3. Nadeau, R.J. et. al. J. Cell. Biochem. 100, 151-160 (2007)

4. Bouchie, A. BioCentury 16(25), A1-A5; June 9, 2008

\section{COMPANIES AND INSTITUTIONS MENTIONED}

American Society of Clinical Oncology, Alexandria, Va. Amgen Inc. (NASDAQ:AMGN), Thousand Oaks, Calif.

Ardea Biosciences Inc. (NASDAQ:RDEA), San Diego, Calif. Aveo Pharmaceuticals Inc., Cambridge, Mass.

Bristol-Myers Squibb Co. (NYSE:BMY), New York, N.Y. California Institute of Technology, Pasadena, Calif.

Dana-Farber Cancer Institute, Boston, Mass.

Eli Lilly and Co. (NYSE:LLY), Indianapolis, Ind.

Harvard Medical School, Boston, Mass.

Howard Hughes Medical Institute, Chevy Chase, Md. ImClone Systems Inc., New York, N.Y.

Johnson \& Johnson (NYSE:JNJ), New Brunswick, N.J.

Mayo Clinic College of Medicine, Rochester, Minn.

Merck KGaA (Xetra:MRK), Darmstadt, Germany

Millennium Pharmaceuticals Inc., Cambridge, Mass.

National Comprehensive Cancer Network, Fort Washington, $\mathrm{Pa}$.

Roche (SWX:ROG), Basel, Switzerland

Takeda Pharmaceutical Co. Ltd. (Tokyo:4502), Osaka, Japan

Trion Pharma GmbH, Munich, Germany 